\title{
Use of end-cutting needles in ultrasound-guided biopsy of neck lesions
}

\author{
H. Y. Yuen • Y. Lee $\cdot$ K. Bhatia $\cdot$ K. T. Wong • \\ A. T. Ahuja
}

Received: 3 July 2011 /Revised: 28 September 2011 / Accepted: 20 October 2011 /Published online: 14 November 2011

(C) European Society of Radiology 2011

\begin{abstract}
The management of a neck mass is dictated by its nature, location and extent. Pathological diagnosis by fine needle aspiration cytology (FNAC) or core biopsy is often required before proceeding to definitive treatment. It is not uncommon for the cytology result to come back as inadequate for various reasons. The unique design of the end-cutting biopsy needle in our experience makes it a good choice for use in obtaining both transcutaneous and intraoral biopsy under ultrasound guidance of neck lesions and serves as a useful alternative or adjunct to FNAC. Although there is, as yet, only a limited evidence base about end-cutting in comparison to side-cutting needles, they carry several potential advantages.

Key Points

- End-cutting needles are safe to use for lesions close to vital structures.

- End-cutting needles yield sufficient tissue samples in a single pass.

- End-cutting biopsy needles are a useful adjunct to FNAC.
\end{abstract}

Keywords Ultrasound · Biopsy · Needle · Neck lesions · Lymph nodes

\section{Introduction}

The management of a neck mass is dictated by its nature, location and extent. While some neck lesions have characteristic clinical signs, ultrasound is superior to

H. Y. Yuen $\cdot$ Y. Lee $\cdot$ K. Bhatia $\cdot$ K. T. Wong • A. T. Ahuja $(\triangle)$

Department of Imaging and Interventional Radiology,

The Chinese University of Hong Kong, Prince of Wales Hospital, 30-32 Ngan Shing Street,

Shatin, New Territories, Hong Kong SAR

e-mail: aniltahuja@cuhk.edu.hk physical examination in sensitivity, specificity and accuracy [1]. Although in most cases a confident diagnosis of neck lesions can be made by ultrasound, pathological confirmation by fine needle aspiration cytology (FNAC) or core biopsy is often required before proceeding to definitive treatment. This article discusses the efficacy of end-cutting core biopsy needle under ultrasound guidance in obtaining core tissue samples for histopathological diagnosis.

\section{Fine needle aspiration cytology vs. core biopsy}

Fine needle aspiration cytology (FNAC) under ultrasound guidance is widely used to supplement diagnostic ultrasound in the assessment of head and neck lesions. It is readily available, inexpensive, relatively non-invasive, well-tolerated and a quick outpatient procedure with a reported diagnostic accuracy in malignant lymphadenopathy that exceeds $90 \%$ [2]. Drawbacks of FNAC include a high proportion of nondiagnostic samples (because of suboptimal smear preparation, scanty aspirate or blood contamination) and incomplete classification especially in cases of lymphoma.

In most institutes the work-up protocol for a neck mass will start with ultrasound and FNAC, which if adequate can proceed to management. However, it is not uncommon for the cytology result to come back as inadequate for the reasons listed above. The non-diagnostic rate for FNAC in head and neck lesions varies from 10 to $30 \%$ [3-6], and is dependent on the expertise of the cytologist. This raises the dilemma of whether to repeat the FNAC or to pursue other methods of obtaining tissue diagnosis. Repeating the FNAC may not always provide the answer, while adding to the patient's anxiety and frustration. Although excision biopsy invariably provides the histological diagnosis, it is an invasive and expensive procedure that is not without risk. Under these circumstances, core needle biopsy under 
ultrasound guidance may be considered a valuable alternative. Moreover, depending on local experience and the level of cytopathological expertise, some institutions adopt cutting needle biopsy rather than FNAC as the preferred approach to tissue sampling.

Core biopsy provides larger tissue samples with preserved tissue architecture, which, together with various immunohistochemical stains, provides a precise histopathological analysis. Cutting needle biopsy is widely accepted as a safe and effective technique for obtaining adequate tissue for the histopathological assessment of head and neck lesions [7-11], including lymph nodes [12,13], salivary glands [14] and thyroid gland [15-18].

Kim et al. [12] reported sensitivity, specificity and accuracy of $97.9 \%, 99.1 \%$ and $97.9 \%$, respectively, for ultrasound-guided core needle biopsy of cervical lymphadenopathy in patients with no known malignancy, and with no procedure-related complications. For ultrasoundguided core biopsies of cervicofacial lymphadenopathy, Screaton et al. [13] reported sensitivity, specificity and accuracy of $98.1 \%, 100 \%$ and $98.7 \%$, respectively, in differentiating benign from malignant lymphadenopathy. Sensitivity, specificity and accuracy in differentiating lymphoma from reactive lymphadenopathy were $98.5 \%$, $100 \%$ and $98.7 \%$, respectively [13]. Histological subclassification of lymphoma was sufficient in $80 \%$ to guide treatment without the need for surgical biopsy [13].

Buckland et al. [14] reported a series of ultrasoundguided cutting-needle biopsy of parotid lesions in 16 patients in which the biopsy provided diagnostic specimens in all patients, and was helpful where FNAC had been inconclusive in 13 of the patients. Diagnostic accuracy of $100 \%$ was obtained in the seven patients who underwent subsequent surgery while the other nine patients avoided unnecessary surgery.

Fine needle aspiration cytology of thyroid nodules offers a rapid, low cost, and usually effective technique for use as a first-line procedure in accordance with general recommendations [15]. Despite the benefit of ultrasound guidance, samples obtained from FNAC of thyroid nodules are inadequate for diagnosis in $15-20 \%$ of patients [16]. Strauss et al. [17] proposed the use of a modified coaxial system for simultaneous FNAC and core biopsy of thyroid nodules when FNAC alone has failed to yield tissue adequate for diagnosis. Kwak et al. [18] advocated the use of ultrasound-guided core needle biopsy as a safe and accurate method for diagnosing thyroid lymphoma and may be a suitable replacement for diagnostic thyroid surgery.

The performance of different biopsy techniques reported in terms of sensitivity, specificity and accuracy should be viewed with caution as different statistical methods may have been employed in analysing the non-diagnostic and inadequate results. In fact, the final evaluation is a combined assessment of both radiological (sampling technique) and pathological (histological interpretation) expertise.

\section{Biopsy needle characteristics: side-cutting needles vs end-cutting needles}

Core biopsy needles in general come under two main categories: side-cutting and end-cutting needles.

Side-cutting needles may be automated or manually operated. These needles consist of two parts, the outer cutting shaft and an inner stylet with a specimen notch. Under ultrasound guidance the needle tip is introduced to the edge of the target tissue, the inner stylet is then advanced into the target tissue with part of the tissue prolapsing into the specimen notch. The specimen is obtained by advancement of the outer cutting shaft to resheath the inner stylet and cut out the specimen core. Removal of the specimen is by simple dislodgement of the sample from the specimen notch.

There are two major disadvantages with the side-cutting needles. The first is that the part of the stylet (typically about $0.5 \mathrm{~cm}$ ) projecting beyond the specimen notch and outer shaft may have to protrude outside the target to accommodate the specimen notch in the optimal position for small lesions, in which case there is an increased risk of jeopardising adjacent structures (Fig. 1). This is a frequent concern for biopsy of neck lesions, many of which are very close to the major neck vessels.

Second, with each pass the amount of sample retrieved is limited by the size of the specimen notch; as a result multiple punctures and passes have to be performed to obtain adequate tissue samples. This is often the case when both histopathology and microbiology or even biochemical tests of the specimens are anticipated.

Modifications in terms of the length of the stylet distal to the specimen notch and the forward throw of the needle (and thus the specimen length) are available with some

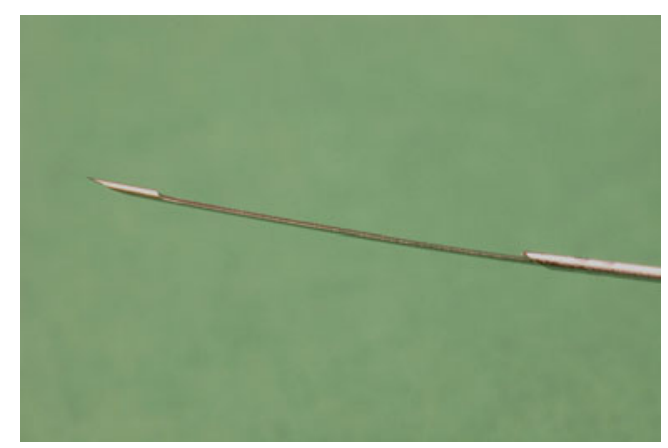

Fig. 1 The inner stylet of the side-cutting needle with the specimen notch. Note that the part of the stylet beyond the specimen notch, which may have to protrude outside the target for optimal positioning, potentially increases the risk of injury to the adjacent structures 
models of side-cutting needles [19] but are not complete remedies.

The end-cutting needles consist of an inner stylet and an outer trochar (Fig. 2). Under ultrasound guidance the needle is introduced such that the needle tip is positioned within the centre of the target. The inner stylet is then removed and the trochar is attached via a connection tube to a syringe. The specimen is obtained by to-and-fro and rotatory movements of the stylet under real-time ultrasound guidance while suction is applied by the syringe (Fig. 3). Removal of the specimen from the needle is by reintroduction of the trochar or saline flush into the specimen bottle.

Operation of the end-cutting needle is straightforward and very similar to the use of fine needles for aspiration cytology. As long as the needle tract is clear and the cutting needle tip is kept within the target tissue it can be safely used for lesions in close proximity to vital structures irrespective of the size of the lesions. Unlike side-cutting needles, no part of the stylet or the sampling area is outside the target tissue even in small lesions. Moreover, the endcutting needle is usually able to obtain sufficient tissue samples in a single pass as long cores of tissue are retrieved into the needle lumen by the cutting and suction mechanism without being limited by the size of the specimen notch as in side-cutting needles (Fig. 4).
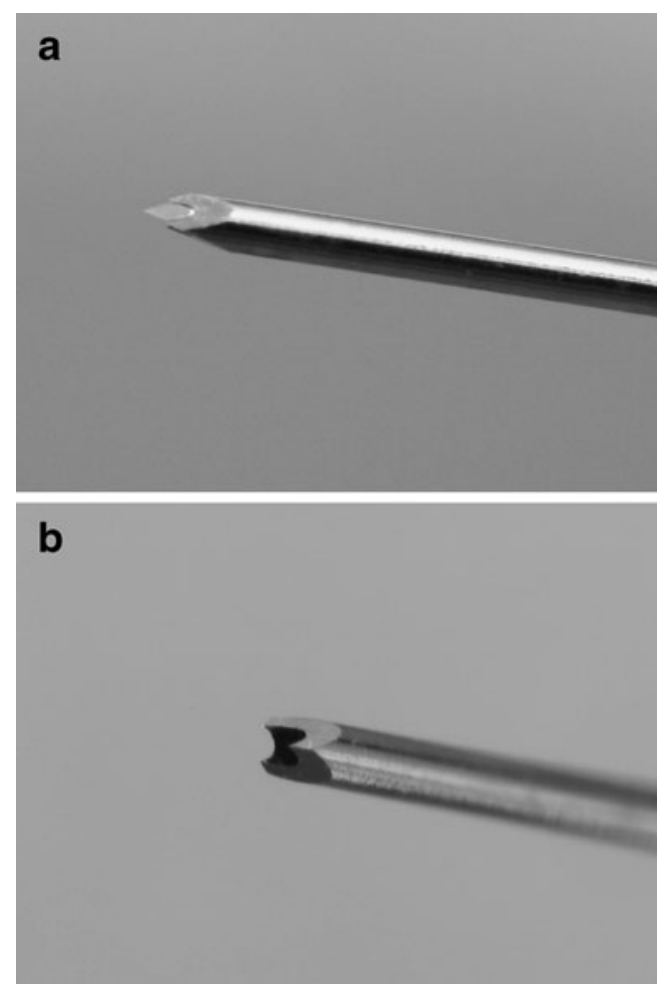

Fig. 2 a The tip of the Franseen type of end-cutting needle with the inner stylet in situ inside the outer trochar. b The serrated tip of the outer trochar after removal of the inner stylet, which is specially designed for cutting to obtain better specimens

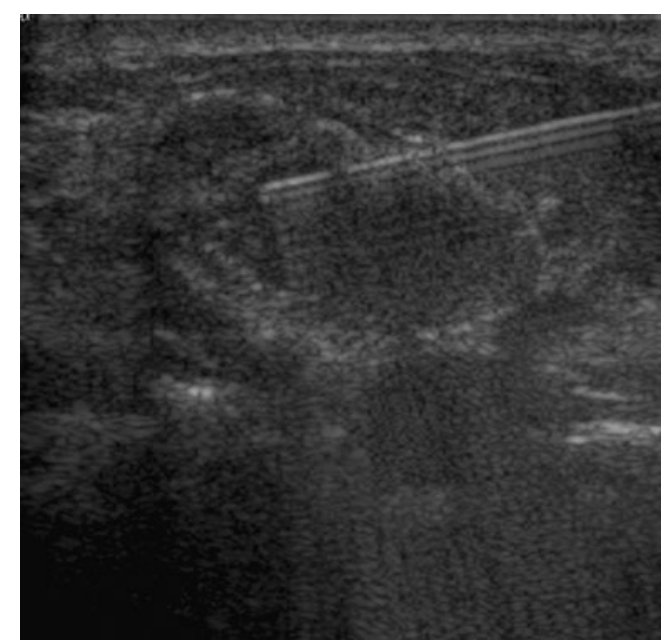

Fig. 3 The tip of the end-cutting needle is inserted under ultrasound guidance into the centre of the target lesion. After removal of the inner stylet and connection of the trochar to a syringe, the specimen is obtained by to-and-fro and rotatory movements of the stylet under real-time ultrasound guidance and syringe suction

There are a number of different end-cutting biopsy needles to choose from, which vary in the design of the tip configuration, in addition to the calibre and length. It has been proposed that needles with a larger bore, certain cutting tips, especially the Franseen trephine type (Angiotech, Medical Device Technologies, USA) and the slotted type and more acute bevel angle tend to produce better specimens [20].

While there are published results on the excellent performance of side-cutting needles for head and neck lesions as stated above, to our knowledge no such data is available for end-cutting needles in this respect. As such direct comparison between the two needles types is not feasible. The use of end-cutting needles for lesions in other body parts is also not evaluated, except for prostatic lesions in which end-cutting needles with increased stroke length tend to increase the mean core length with less fragmentation but are associated with higher capture failure rate and

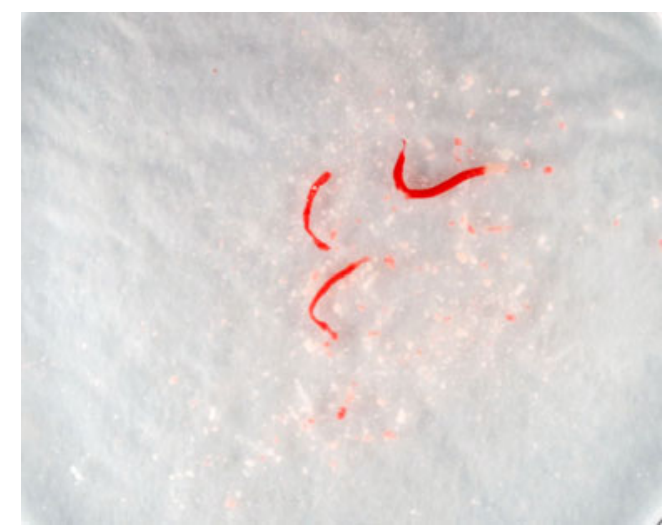

Fig. 4 Using the end-cutting needle, sufficient tissue samples are usually obtained in a single pass as long cores of tissues are retrieved into the needle lumen by the cutting and suction mechanisms 
slightly greater morbidity (pain and possibly fever) and no advantage in capsular coverage [21]. However, the different clinical context makes inference for the use in head and neck lesions difficult.

Our own experience in using the Franseen trephine type needle with the serrated stylet tip showed that it is safe and provides a very high yield in terms of adequacy and accuracy. This approach is simply an extension of the technique of ultrasound-guided FNAC by using an appropriately sized end-cutting needle for obtaining biopsy samples rather than cytology. The cutting action at the needle tip only occurs while it is within the lesion and under real-time ultrasound guidance. The risk of the needle hitting normal structures that are occult on ultrasound, such as the facial nerve within the parotid gland, during advancement of the needle with the trochar in situ is minimal and no greater than that of FNAC. No complication of tumour seeding along the biopsy tract, induction of scarring or significant haemorrhage had been encountered, presumably because of the small calibre of the needles used and the small number (often only one) of needle passes required. Biopsies using these end-cutting needles in our experience provide definitive diagnosis, despite preceding inconclusive FNAC results. Feedback from colleagues specialising in head and neck pathology in our institute was positive in that specimens obtained by these end-cutting needles tend to be copious. Although such specimens may be more fragmented than those obtained by side-cutting needles, this does not degrade the diagnostic quality. However, one should cautiously refrain from using excessive suction during biopsy so as to avoid heavily blood-stained tissue samples.

In addition to the usual application in biopsy of lymphadenopathy, salivary gland lesions and thyroid lesions, we have further extended the use of the Franseen needle to biopsy of deep-seated head and neck lesions under intraoral ultrasound guidance [22]. The reduced risk of injury to adjacent vessels and the high yield in a single pass by using the Franseen needle compared with the use of the side-cutting needles as described above make it ideal for this purpose.

In general $18 \mathrm{G}$ or $20 \mathrm{G}$ Franseen needles are adequate for biopsy of lymph nodes and salivary gland lesions while $22 \mathrm{G}$ Franseen needles are reserved for biopsy of smaller lesions and in the biopsy of deep-seated lesions via the intraoral approach. The needle length of $9 \mathrm{~cm}$ is easier to manipulate and optimal for transcutaneous biopsy of neck lesions as these are mostly superficial in location. $15-\mathrm{cm}$ or $20-\mathrm{cm}$ long needles are also available and are more suitable for the intraoral approach because of the limited space within the oral cavity with the transducer in situ. Local anaesthetic is used to reduce pain and the use of local anaesthetic spray in the intraoral route also reduces the gag reflex. A coagulation screen is not routinely performed unless there is clinical concern regarding coagulopathy, and the procedure can be safely performed on an outpatient basis.

\section{Conclusion}

The unique design of the Franseen trephine needle in our experience makes it a good choice for use in obtaining both transcutaneous and intraoral biopsy under ultrasound guidance of neck lesions and serves as a useful alternative or adjunct to fine needle aspiration cytology. Although there is, as yet, only a limited evidence base about end-cutting in comparison to side-cutting needles, they carry several potential advantages. In particular they should be considered when FNAC fails to provide definitive diagnosis for thyroid lesions and thyroid lymphoma is suspected, or when suspicious parapharyngeal lesions cannot be safely accessed by other means.

\section{References}

1. Adeyemo WL, Ogunlewe MO, Ladeinde AL (2006) Ultrasound as a diagnostic aid in head and neck lesions. Niger Postgrad Med J $13: 147-152$

2. Kline TS, Kannan V, Kline IK (1984) Lymphadenopathy and aspiration biopsy cytology: review of 376 superficial nodes. Cancer 54:1076-1081

3. Schelkun P, Grundy W (1991) Fine-needle aspiration biopsy of head and neck lesions. J Oral Maxillofac Surg 49:262-267

4. Chow LS, Gharib H, Goellner JR, Van Heerden JA (2001) Nondiagnostic thyroid fine-needle aspiration cytology: management dilemmas. Thyroid 11:1147-1151

5. Alexander EK, Heering JP, Benson CB, Frates MC, Doubilet PM, Cibas ES, Marqusee E (2002) Assessment of nondiagnostic ultrasound-guided fine needle aspirations of thyroid nodules. J Clin Endocrinol Metab 87:4924-4927

6. Rakhshan M, Rakhshan A (2009) The diagnostic accuracy of fine needle aspiration cytology in neck lymphoid masses. Iran J Pathol 4:147-150

7. Yamashita Y, Kurokawa H, Takeda S, Fukuyama H, Takahashi T (2002) Preoperative histologic assessment of head and neck lesions using cutting needle biopsy. Oral Surg Oral Med Oral Pathol Oral Radiol Endod 93:528-533

8. Kraft M, Gurtler N, Schmuziger N, Arnoux A (2007) Ultrasoundguided core-needle biopsy in the diagnosis of head and neck lesions. J Laryngol Otol 121:895-896

9. Ridder GJ, Pfeiffer J (2007) Usefulness of cutting needle biopsy in recurrent and advanced staged head and neck malignancies in a palliative setting. Support Care Cancer 15:1301-1307

10. Nyquist GG, Tom WD, Mui S (2008) Automatic core needle biopsy - a diagnostic option for head and neck masses. Arch Otolaryngol Head Neck Surg 134:184-189

11. Bearcroft P, Berman L, Grant J (2009) The use of ultrasoundguided cutting-needle biopsy in the neck. Clin Radiol 50:690-695

12. Kim BM, Kim EK, Kim MJ, Yang WI, Park CS, Park SI (2007) Sonographically guided core needle biopsy of cervical lymphadenopathy in patients without known malignancy. J Ultrasound Med 26:585-591 
13. Screaton NJ, Berman LH, Grant JW (2002) Head and neck lymphadenopathy: evaluation with US-guided cutting-needle biopsy. Radiology 224:75-81

14. Buckland JR, Manjaly G, Violaris N, Howlett DC (1999) Ultrasound-guided cutting-needle biopsy of the parotid gland. J Laryngol Otol 113:988-992

15. Frates MC, Benson CB, Charboneau JW et al (2005) Society of radiologists in ultrasound. Management of thyroid nodules detected at US: Society of radiologists in ultrasound consensus conference statement. Radiology 237:794-800

16. Alexander EK, Heering JP, Benson CB et al (2002) Assessment of nondiagnostic ultrasound-guided fine needle aspirations of thyroid nodules. J Clin Endocrinol Metab 87:4924-4927

17. Strauss EB, Iovino A, Upender S (2008) Simultaneous fine-needle aspiration and core biopsy of thyroid nodules and other superficial head and neck masses using sonographic guidance. AJR 190:1697-1699
18. Kwak JY, Kim EK, Ko KH, Yang WI, Kim MJ, Son EJ, Oh KK, Kim KW (2007) Primary thyroid lymphoma-role of ultrasound-guided needle biopsy. J Ultrasound Med 26:17611765

19. Berman L (2000) Core biopsy. In: Practical head and neck ultrasound. Greenwich Medical Media/Cambridge University Press, Cambridge

20. Andriole JG, Hagga JR, Adams RB, Nunez C (1983) Biopsy needle characteristics assessed in the laboratory. Radiology 148:659-662

21. Ubhayakar GN, Li WY, Corbishley CM, Patel U (2002) Improving glandular coverage during prostate biopsy using a long-core needle: technical performance of an end-cutting needle. BJU Int 89:40-43

22. Wong KT, Tsang RKY, Tse GMK, Yuen EHY, Ahuja AT (2006) Biopsy of deep-seated head and neck lesions under intraoral ultrasound guidance. Am J Neuroradiol 27:1654-1657 